\title{
Seroma em lipoabdominoplastia e abdominoplastia: estudo ultrassonográfico comparativo
}

\author{
Seroma in lipoabdominoplasty and abdominoplasty: ultrasonographic \\ comparative study
}

\author{
Marcello Di Martino ${ }^{1}$ \\ FÁbio XeRFAn NAHAS ${ }^{2}$ \\ NeIl Ferreira Novo ${ }^{3}$ \\ Alexandro Kenji Kimura ${ }^{4}$ \\ Lydia Masako Ferreira 5
}

Trabalho realizado na Universidade Federal de São Paulo - Escola Paulista de Medicina, Disciplina de Cirurgia Plástica, São Paulo, SP, Brasil.

Trabalho recebeu menção honrosa do Prêmio Nemer Chidid 2010

Artigo submetido no SGP (Sistema de Gestão de Publicações) da RBCP.

Artigo recebido: $24 / 7 / 2010$ Artigo aceito: $25 / 11 / 2010$

\begin{abstract}
RESUMO
Introdução: O seroma é uma das complicações mais frequentes nas abdominoplastias. Objetivo: Comparar a incidência de seroma em pacientes submetidos à abdominoplastia convencional e à lipoabdominoplastia. Método: Foram estudadas 41 pacientes, divididas em dois grupos: Grupo A (21 pacientes submetidas à abdominoplastia convencional e Grupo B (20 pacientes submetidas à lipoabdominoplastia). Para a investigação da formação de seroma foi realizado ultrassonografia de cinco regiões da parede abdominal (epigástrio (EPI), umbilical (UMB), hipogástrio (HIPO), fossa ilíaca direita (FID) e fossa ilíaca esquerda (FIE)) em dois momentos: entre o $11^{\circ}$ e o $14^{\circ}$ dia de pós-operatório (DPO) (P1) e entre o $18^{\circ}$ e $21^{\circ}$ DPO (P2). Resultados: A incidência de seroma tanto em P1 quanto em P2 foi significativamente maior no grupo A que no grupo B. Observou-se que no grupo A, no P1, as regiões FID e FIE apresentaram maior incidência de coleções fluidas. No grupo B, houve uma maior incidência de coleções fluidas na região HIPO em P1 e nas regiões UMB e HIPO em P2. Conclusão: Houve menor incidência de seroma nos pacientes submetidos à lipoabdominoplastia em relação à abdominoplastia convencional nos momentos estudados.
\end{abstract}

Descritores: Seroma. Abdome/cirurgia. Lipectomia. Cirurgia plástica. Ultrassonografia.

\begin{abstract}
Background: Seroma is one of the most common complications in abdominoplasty. Objective: To compare the rate of seroma formation in patients who underwent either abdominoplasty and lipoabdominoplasty. Methods: The sample consisted of 41 female patients, who were divided into three groups and underwent one of the following procedures: group $A(n=21)$, abdominoplasty and group $B(n=20)$, lipoabdominoplasty. In order to investigate seroma formation, abdominal ultrasound was performed in 5 regions of the abdominal wall [epigastrium (EPI), umbilical (UMB), hypogastrium (HYPO), right iliac fossa (RIF) and left iliac fossa (LIF)], at two postoperative periods: (P1), between postoperative days 11 and 14, and (P2), between postoperative days 18 and 21. Results: The rate of seroma formation at both $\mathrm{P} 1$ and $\mathrm{P} 2$ was significantly higher in group A than in group B. It was observed that, in group $\mathrm{A}$ at $\mathrm{P} 1$, the regions RIF and LIF developed larger fluid collections. In group B, there were significantly larger fluid collections in the HYPO region at P1, and in the UMB and HYPO regions at P2. Conclusion: Lipoabdominoplasty is effective technique for the prevention of seroma compared with abdominoplasty.
\end{abstract}

Keywords: Seroma. Abdomen/surgery. Lipectomy. Plastic surgery. Ultrasonography.

\footnotetext{
1. Mestre em Cirurgia Plástica, Membro Titular da SBCP.

2. Livre docência e Pós-Doutorado; Professor afiliado da Disciplina de Cirurgia Plástica da UNIFESP-EPM, Responsável pelo Setor de Deformidades da Parede Abdominal da Disciplina de Cirurgia Plástica da UNIFESP-EPM, Membro Titular da SBCP.

3. Professor Titular da Disciplina de Saúde Pública da Universidade de Santo Amaro.

4. Mestrado, Especialista em Cirurgia Plástica pela SBCP.

5. Professora Titular da Disciplina de Cirurgia Plástica UNIFESP-EPM; Livre docência e Pós-Doutorado; Chefe do Departamento de Cirurgia da UNIFESP-EPM; Coordenadora do Programa Pós-Graduação em Cirurgia Plástica UNIFESP-EPM; Membro titular da SBCP.
} 


\section{INTRODUÇÃO}

A abdominoplastia é um dos procedimentos mais realizadas em todo mundo, sendo a terceira cirurgia estética mais realizada no Brasil, em 2008'. As complicações locais (seroma, hematoma, necrose do retalho dermogorduroso) são as mais frequentes, sendo que complicações sistêmicas, como trombose venosa profunda e embolia pulmonar, são raras ${ }^{2}$.

O seroma pode ser definido como uma coleção líquida, de características exsudativas, formada profundamente ao retalho dermogorduroso ${ }^{3,4}$. É a complicação precoce mais frequente nas abdominoplastias com incidência que varia de 1 a $57 \%$, sendo $10 \%$ a média aceita pela maioria dos autores ${ }^{5,6}$. A ultrassonografia tem sido o método de escolha para o diagnóstico de seroma pós abdominoplastia.

Para reduzir o alto índice de seroma no pós-operatório algumas medidas têm sido preconizadas, como manipulação reduzida do retalho cutâneo, menor tempo operatório, uso de drenos de sucção e uso de malhas de compressão no pósoperatório durante 30 dias $^{7}$. Baroudi \& Ferreira ${ }^{3}$ descreveram os pontos de fixação do retalho ("quilting sutures") com a finalidade de minimizar o espaço morto e, consequentemente, diminuir o acúmulo de líquido.

A lipoaspiração, introduzida por Illouz ${ }^{8}$, revolucionou o tratamento das lipodistrofias, particularmente da região abdominal. As décadas de 80 e 90 foram marcadas por uma associação cada vez mais frequente entre lipoaspiração e abdominoplastia ${ }^{9-11}$, acompanhada também de aumento no número de complicações, entre elas necrose cutânea, epiteliólise, deiscências, hematoma e, principalmente, seroma ${ }^{12}$.

$\mathrm{Na}$ tentativa de minimizar os riscos de complicação, Saldanha et al. ${ }^{13,14}$ descreveram a lipoabdominoplastia, procedimento no qual se associa a lipoaspiração à abdominoplastia com descolamento reduzido e preservação da fáscia de Scarpa na região infraumbilical. Entretanto, estudos são controversos ao analisar se a lipoaspiração associada à abdominoplastia aumentaria a incidência de seroma ${ }^{12,15-17}$. Além disso, não existe nenhum estudo na literatura que tenha comparado a ocorrência de seroma na abdominoplastia e na lipoabdominoplastia por meio de análise com ultrassom.

Portanto, o objetivo desse estudo é comparar a ocorrência de seroma em pacientes submetidos à abdominoplastia convencional e a lipoabdominoplastia.

\section{MÉTODO}

Este é um estudo clínico, prospectivo, realizado entre janeiro de 2006 e março de 2009, com um grupo de 41 pacientes do gênero feminino. Após aprovação pelo comitê de ética em pesquisa e assinatura do termo de consentimento livre e esclarecido, as pacientes foram distribuídas por ordem de chegada em dois grupos: as primeiras 21 pacientes foram submetidas à abdominoplastia convencional (grupo A) e as 20 pacientes seguintes foram submetidas à lipoabdominoplastia (grupo B).

Os critérios de inclusão foram idade entre 25 e 60 anos, deformidade de pele e tela subcutânea da região abdominal tipo III de Nahas $^{18}$ (na qual foi indicada a remoção de toda a pele e tela subcutânea entre a cicatriz umbilical e a região suprapúbica), deformidade músculo-aponeurótica tipo A de Nahas ${ }^{19}$ (na qual está indicada a plicatura da lâmina anterior da bainha do músculo reto do abdome para correção da diástase) e índice de massa corpórea (IMC) menor ou igual a $30 \mathrm{~kg} / \mathrm{m}^{2}$.

Os critérios de exclusão foram tabagismo, lipoaspiração ou outra cirurgia abdominal prévia, cicatriz na parede abdominal (exceto Pfannestiel), hérnia de parede abdominal, antecedentes de trombose venosa profunda, doenças do sistema linfático, doença pulmonar obstrutiva crônica, hepatopatia, neoplasia, uso de medicação antidepressiva, hipertensão e diabetes ou outras doenças sistêmicas crônicas e pacientes com grandes perdas de peso.

No grupo A, a idade variou de 26 a 50 anos (média de 34,8 anos) e o índice de massa corpórea (IMC) entre 20 e 28 $\mathrm{kg} / \mathrm{m}^{2}$ (média de $23,7 \mathrm{~kg} / \mathrm{m}^{2}$ ) e, no grupo $\mathrm{B}$, a idade variou de 26 a 53 anos (média de 34,9 anos) e o IMC entre 21,3 a $29,6 \mathrm{~kg} / \mathrm{m}^{2}$ (média de $25,1 \mathrm{~kg} / \mathrm{m}^{2}$ ).

\section{Técnica operatória}

Em todos os grupos, as pacientes foram submetidas à anestesia geral:

- Grupo A (n=21) - Abdominoplastia. Foi realizada incisão na região suprapúbica, prolongando-se lateralmente até as cristas ilíacas em área previamente demarcada. O descolamento do retalho dermogorduroso de sua aponeurose muscular estendeu-se superiormente até $2 \mathrm{~cm}$ inferiormente ao apêndice xifoide $\mathrm{e} 3 \mathrm{~cm}$ lateralmente às margens mediais dos músculos retos abdominais (Figura 1). A diástase foi corrigida pela plicatura da lâmina anterior da bainha dos músculos retos abdominais, em plano único, com pontos simples invertidos, utilizando-se fio de náilon monofilamentar 2-0. Não foi realizada lipoaspiração.

- Grupo B (n=20) - Lipoabdominoplastia. Foi realizada infiltração de toda região abdominal previamente demarcada com solução fisiológica associada à adrenalina na proporção de 1:500.000 UI, sendo que a quantidade de solução infiltrada foi igual a quantidade de gordura aspirada (técnica superúmida 1:1). A lipoaspiração foi iniciada pela região supraumbilical e flancos com cânula de $3 \mathrm{~mm}$ de espessura, seguida pela região infraumbilical com cânula de $4 \mathrm{~mm}$ de espessura. (Figura 2). O total lipoaspirado variou entre 600 a $2.700 \mathrm{ml}$ (média de $1.327 \mathrm{ml}$ ). Em seguida foi realizada incisão suprapúbica prolongando-se até as cristas ilíacas em área previamente demarcada. 


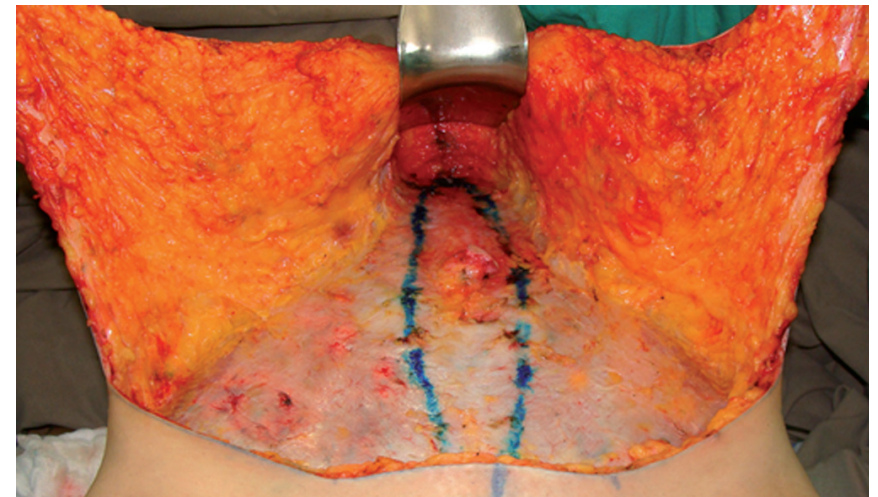

Figura 1 - Grupo A - Abdominoplastia convencional. Área de descolamento do retalho dermogorduroso, no plano supraaponeurótico, estendendo-se $3 \mathrm{~cm}$ lateralmente às margens mediais dos músculos retos do abdome até o processo xifoide.

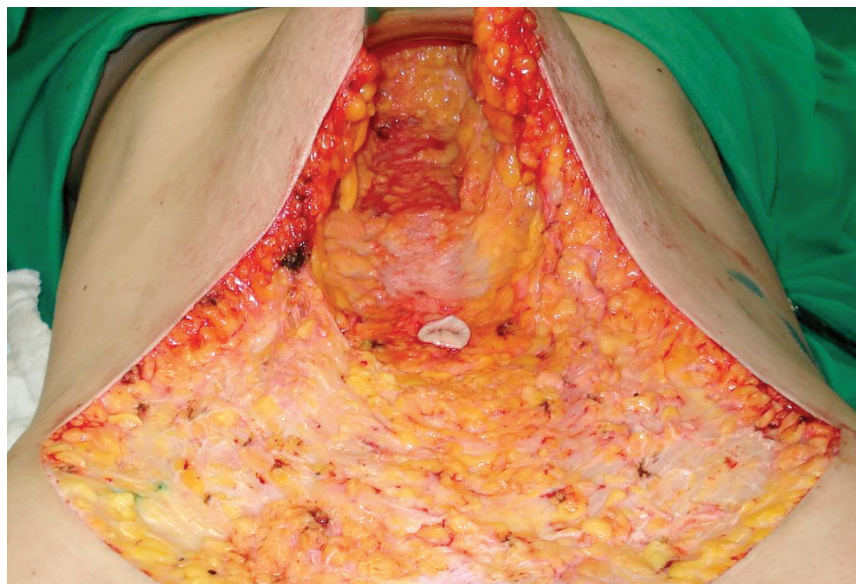

Figura 3 - Demonstração do descolamento na região supraumbilical que foi realizado na linha média, estendendo-se $1 \mathrm{~cm}$ lateralmente às margens mediais dos músculos retos do abdome, até o processo xifoide.

O descolamento do retalho dermogorduroso foi realizado anteriormente à fáscia de Scarpa até a região umbilical. $\mathrm{Na}$ região supraumbilical, foi realizado um descolamento 1 $\mathrm{cm}$ lateralmente às margens mediais dos músculos retos do abdome, até o apêndice xifoide. Na linha mediana infraumbilical, foi removido um fuso vertical, contendo a fáscia de Scarpa e tecido gorduroso, para expor as margens mediais dos músculos retos do abdome (Figura 3).

Foi realizada a plicatura da lâmina anterior da bainha dos músculos retos do abdome em plano único com fio de náilon monofilamentar 2-0, utilizando-se pontos separados invertidos.

Nos dois grupos, o descolamento foi realizado com eletrocautério com potência de 35 watts para dissecção e 35
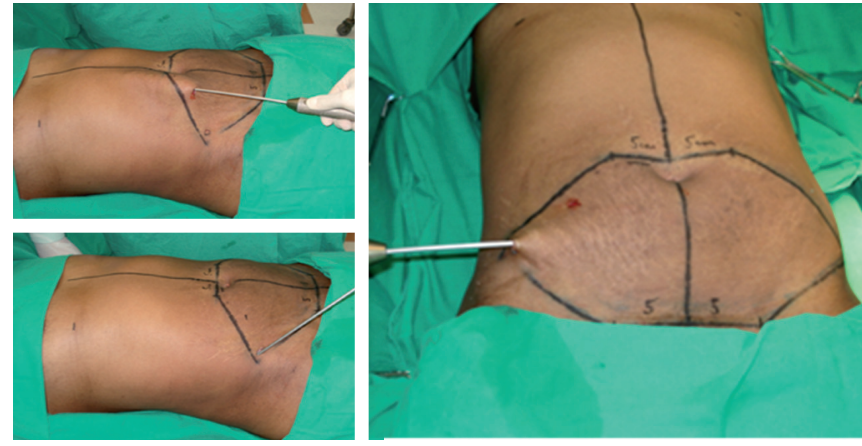

Figura 2 - Lipoaspiração iniciando-se pela região supraumbilical (A) no plano profundo continuando-se nos flancos (B) com cânula de 3,5 $\mathrm{mm}$ de espessura. Na região infraumbilical $(\boldsymbol{C})$, iniciou-se pela lipoaspiração no plano profundo com cânula de 3,5 $\mathrm{mm}$ de espessura, seguida pela lipoaspiração no plano superficial com cânula de 4,0 $\mathrm{mm}$ de espessura.

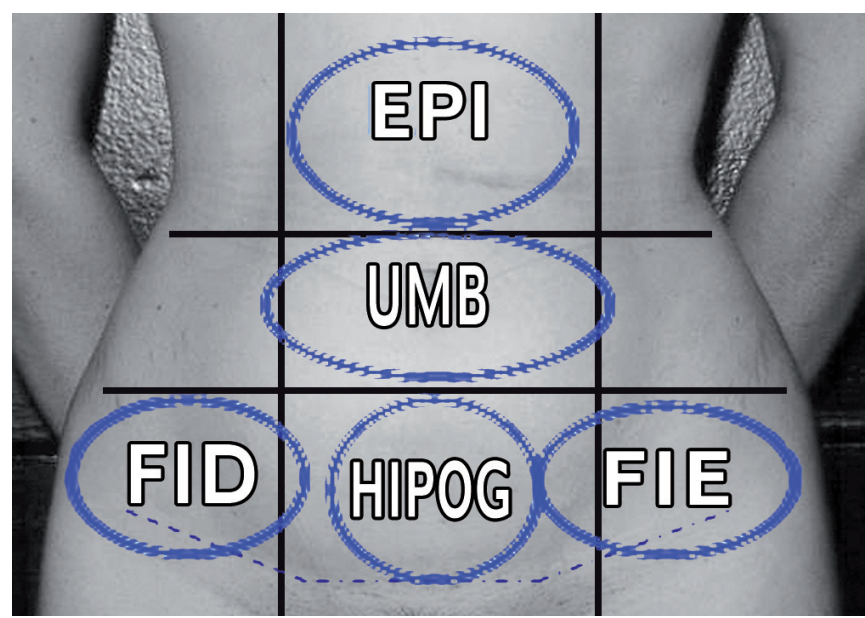

Figura 4-Áreas a serem submetidas ao exame de ultrassonografia para identificação de seroma. EPI: epigástrio, UMB: umbilical, FID: fossa iliaca direita, HIPO: hipogástrio e FIE: fossa ilíaca esquerda.

watts para coagulação. O pedículo do umbigo foi fixado à aponeurose muscular com pontos simples de náilon monofilamentar 4-0.

Dreno de sucção foi utilizado em todos os pacientes, nos dois grupos. Dois drenos com diâmetro de 3,2 mm (Portovac, Kalmédica, Campinas, Brasil) foram posicionados sob o retalho abdominal e exteriorizados na região suprapúbica e mantidos até que ocorresse um débito menor que $40 \mathrm{ml}$ em 24 horas.

As pacientes foram orientadas a usar malha abdominal compressiva pelo período de 30 dias e a permanecer em posição de Fowler por 10 dias. O período de internação foi de 24 horas em todos os casos e a deambulação precoce foi estimulada no primeiro dia de pós-operatório. Não foi realizada drenagem linfática. 


\section{Avaliação ultrassonográfica}

As pacientes foram submetidas a acompanhamento ultrassonográfico em dois períodos: entre o $11^{\circ}$ e o $14^{\circ} \mathrm{PO}$ (P1) e entre o $18^{\circ}$ e $21^{\circ} \mathrm{PO}(\mathrm{P} 2)$. O exame ultrassonográfico foi executado pelo mesmo examinador, realizando varreduras longitudinais, oblíquas e transversais, em cinco regiões da parede abdominal. Estas regiões foram obtidas pela intersecção de 2 linhas horizontais e duas verticais, sendo a primeira linha transversal situada $3 \mathrm{~cm}$ superiormente à cicatriz umbilical e a segunda linha, $3 \mathrm{~cm}$ inferiormente à cicatriz umbilical. As 2 linhas verticais passam pelo ponto médio dos ligamentos inguinais, definindo assim as regiões: epigástrica (epi), umbilical (umb), hipogástrica (hipo), fossa ilíaca direita (FID) e fossa ilíaca esquerda (FIE) (Figura 4).

$\mathrm{O}$ exame foi realizado com a paciente em decúbito dorsal horizontal, mensurando-se o volume em mililitros $(\mathrm{ml})$. Nos casos em que a coleção líquida por região ultrapassou $20 \mathrm{ml}$, realizou-se punção esvaziadora guiada pelo ultrassom. Os casos que apresentaram volume maior que $20 \mathrm{ml}$ somando-se as cinco regiões, foram considerados positivos para seroma.

\section{Análise estatística}

A análise estatística foi realizada por meio de testes não paramétricos, levando-se em consideração a natureza das variáveis estudadas. O teste de Mann-Whitney foi aplicado com o objetivo de comparar os grupos A e B em relação a idade, número de dias com dreno e IMC. O mesmo teste foi aplicado para comparar os dois grupos em relação ao volume de coleções fluidas. Tal comparação foi realizada em separado para os períodos 11-14 (P1) e 18-21 dias (P2).

$\mathrm{O}$ teste exato de Fisher foi aplicado com a finalidade de comparar os grupos A e B em relação aos achados positivos para seroma, em separado para os períodos P1 e P2. Este teste foi aplicado também para comparar as complicações pós-operatórias apresentadas nos grupos A e B.

$\mathrm{O}$ teste de Wilcoxon foi aplicado com o propósito de comparar, em separado para os grupos A e B, os volumes totais de coleções fluidas observados nos períodos P1 e P2.

$\mathrm{O}$ teste de McNemar foi aplicado para comparar os períodos P1 e P2 em relação aos achados positivos para seroma, separadamente para os grupos A e B. Este teste foi aplicado também para comparar o diagnóstico clínico e ultrassonográfico de seroma nos grupos A e B, no período P1.

$O$ teste $G$ de Cochran foi utilizado para comparar a presença de coleções fluidas em cada uma das cinco regiões, nos dois grupos, nos dois períodos.

O nível de rejeição da hipótese de nulidade foi fixado em 0,05 ou $5 \%(\mathrm{p} \leq 0,05)$.

\section{RESULTADOS}

Não houve diferença estatística em relação ao IMC e idade nos dois grupos. Em relação ao tempo de permanência do dreno, houve diferença estatística significante, sendo maior no grupo A (média de 4,38 dias) do que no grupo B (média de 2,95 dias) (Tabela 1).

Tabela 1 - Diferença entre os grupos A (abdominoplastia) e B (lipoabdominoplastia) em relação ao $\mathrm{IMC}^{1}$, idade e ao tempo de permanência do dreno.

\begin{tabular}{l|c|c|c}
\hline Grupo & IMC & Idade (anos) & Dias com dreno \\
\hline $\mathrm{A}(\mathrm{n}=21)$ & $23,7(20-28)$ & $34,8(26-50)$ & $4,38(3-7)$ \\
$\mathrm{B}(\mathrm{n}=20)$ & $25,1(21,3-29,6)$ & $34,9(26-53)$ & $2,95(2-5)$ \\
\hline Valor de $\mathrm{p}$ & 0,06 & 0,79 & $<0,0006^{*}$ \\
\hline
\end{tabular}

I.M.C. - Índice de massa corpórea; * estatisticamente significante Teste de Mann-Whitney.

Tabela 2 - Comparação entre o grupo A (abdominoplastia) e o grupo B (lipoabdominoplastia) em relação à presença de seroma em P1(11-14 dias) e P2 (18-21 dias).

\begin{tabular}{|c|c|c|c|c|c|c|}
\hline Período & Grupo & \multicolumn{2}{|c|}{ Seroma } & Total & $\%$ com seroma & $\mathbf{p}$ \\
\hline $\begin{array}{l}\mathrm{P} 1 \\
\mathrm{P} 1\end{array}$ & $\begin{array}{c}\text { A } \\
\text { B } \\
\text { Total }\end{array}$ & $\begin{array}{c}8 \\
2 \\
10\end{array}$ & $\begin{array}{l}13 \\
18 \\
31\end{array}$ & $\begin{array}{l}21 \\
20 \\
41\end{array}$ & $\begin{array}{l}38,1 \\
10,0 \\
24,4\end{array}$ & $0,040^{*}$ \\
\hline $\begin{array}{l}\text { P2 } \\
\text { P2 }\end{array}$ & $\begin{array}{c}\text { A } \\
\text { B } \\
\text { Total }\end{array}$ & $\begin{array}{l}7 \\
0 \\
7\end{array}$ & $\begin{array}{l}14 \\
20 \\
34\end{array}$ & $\begin{array}{l}21 \\
20 \\
41\end{array}$ & $\begin{array}{c}33,3 \\
0,0 \\
41,0\end{array}$ & $0,005^{*}$ \\
\hline
\end{tabular}

* Diferença estatisticamente significante $(\mathrm{p} \leq 0,05)$. Teste exato de Fisher. 


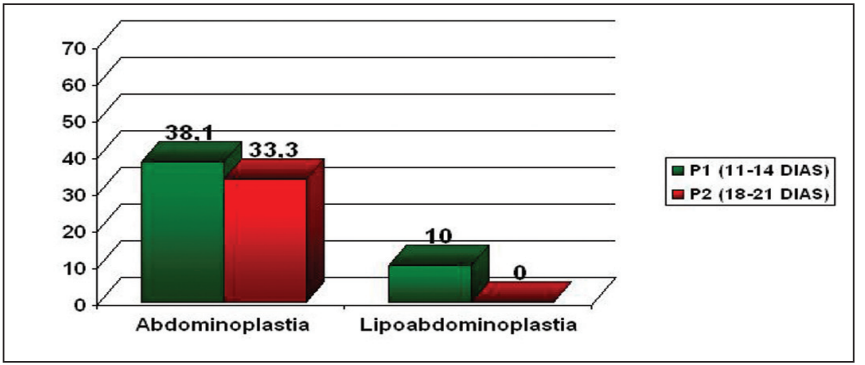

Figura 5 - Diferença nas incidências de seroma nos grupos $A$ (abdominoplastia) e B (lipoabdominoplastia), nos períodos de 11-14 dias e 18-21 dias.

No P1 (11-14 dias), a incidência de seroma no grupo A foi de $38,1 \%$ e, no grupo B, foi de $10 \%$. No P2 (18-21 dias), a incidência de seroma foi de $33,3 \%$, no grupo A, e 0 , no grupo B. O teste exato de Fisher demonstrou que a porcentagem de seroma, tanto no P1 quanto no P2, foi significantemente maior no grupo A (Tabela 2).

$\mathrm{O}$ teste de McNemar demonstrou que não houve diferença entre os períodos P1 e P2 em relação à formação de seroma comparando-se separadamente cada grupo (Figura 5).

Analisando-se o volume de coleções fluidas encontradas, o teste de Wilcoxon demonstrou redução significante entre P1 e P2 no grupo B. No grupo A, não houve diferença significante em relação ao volume de coleções fluidas nos dois períodos (Tabela 3).

Constatou-se pelo teste de Mann-Whitney que não houve diferença significante entre os grupos A e B no P1 (11-14 dias), em relação ao volume de coleções fluidas. No entanto, no P2, houve diferença significante em relação ao volume de coleções fluidas entre os dois grupos, sendo maior no grupo A do que no grupo B (Tabela 4).

No grupo A (abdominoplastia), no período de 11-14 dias (P1), o seroma foi diagnosticado clinicamente em 23,3\%

\begin{tabular}{c|c|c|c|c|c|c|c}
\hline \multicolumn{7}{c}{ Tabela 3 - Comparação do volume de coleções fluidas medidos pela ultrassonografia entre os períodos } \\
P1 (11-14 dias) e P2 (18-21 dias) nos grupos A (abdominoplastia) e B (lipoabdominoplastia). \\
\hline Grupo & N & Período & Média (ml) & Mediana (ml) & Mínimo (ml) & Máximo (mI) & p \\
& & & & & & & \\
\hline A & 21 & P1 & 53,3 & 9,3 & 0 & 576 & N/S \\
& & P2 & 73,1 & 8,9 & 0 & 580 & \\
\hline B & 20 & P1 & 8,4 & 3,0 & 0 & 41,9 & $0,0002^{*}$ \\
& & P2 & 2,1 & 0,2 & 0 & 9,5 & \\
\hline
\end{tabular}

* Diferença estatisticamente significante $(\mathrm{p} \leq 0,05)$. Teste de Wilcoxon.

Tabela 4 - Comparação em relação ao volume de coleções fluidas medidos pela ultrassonografia entre os grupos A (abdominoplastia) e B (lipoabdominoplastia) nos períodos P1 (11-14 dias) e P2 (18-21 dias).

\begin{tabular}{c|c|c|c|c|c|c|c}
\hline Grupo & N & Período & Média $(\mathbf{m l})$ & Mediana $(\mathbf{m l})$ & Mínimo $(\mathbf{m l})$ & Máximo $(\mathbf{m l})$ & P \\
\hline A & 21 & P1 & 53,3 & 9,3 & 0 & 576 & N/S \\
B & 20 & P1 & 8,4 & 3,0 & 0 & 41,9 \\
\hline A & 21 & P2 & 73,1 & 8,9 & 0 & 580 \\
B & 20 & P2 & 2,1 & 0,2 & 0 & 9,5 & $0,020^{*}$ \\
\hline
\end{tabular}

*Diferença estatisticamente significante $(\mathrm{p} \leq 0,05)$. Teste de Mann-Whitney.

Tabela 5 - Comparação entre o diagnóstico clínico e ultrassonográfico de seroma nos grupos A (abdominoplastia) e B (lipoabdominoplastia) no período de 11-14 dias (P1).

\begin{tabular}{|c|c|c|c|c|c|c|}
\hline \multirow[t]{2}{*}{ Grupo } & \multirow[t]{2}{*}{ Diagnóstico } & \multicolumn{2}{|c|}{ Seroma } & \multirow[t]{2}{*}{ Total } & \multirow[t]{2}{*}{$\%$ com seroma } & \multirow[t]{2}{*}{$\mathbf{p}$} \\
\hline & & com & sem & & & \\
\hline Grupo A & $\begin{array}{l}\text { Clínico } \\
\text { Ultrassom }\end{array}$ & $\begin{array}{l}5 \\
8\end{array}$ & $\begin{array}{l}16 \\
13\end{array}$ & $\begin{array}{l}21 \\
21\end{array}$ & $\begin{array}{l}23,3 \\
38,1\end{array}$ & $\leq 0,024^{*}$ \\
\hline Grupo B & $\begin{array}{l}\text { Clínico } \\
\text { Ultrassom }\end{array}$ & $\begin{array}{l}1 \\
2\end{array}$ & $\begin{array}{l}19 \\
18\end{array}$ & $\begin{array}{l}20 \\
20\end{array}$ & $\begin{array}{c}5,0 \% \\
10,0 \%\end{array}$ & $\mathrm{~N} / \mathrm{S}$ \\
\hline
\end{tabular}

* Diferença estatisticamente significante $(\mathrm{p} \leq 0,05)$. Teste de McNemar. 
Tabela 6 - Comparação entre os grupos A (abdominoplastia) e B

(lipoabdominoplastia) em relação às complicações gerais pós-operatórias.

\begin{tabular}{|c|c|c|c|c|c|}
\hline \multirow[t]{2}{*}{ Complicações } & \multicolumn{2}{|c|}{ Grupo A ( $n=21)$} & \multicolumn{2}{|c|}{ Grupo B $(n=20)$} & \multirow[t]{2}{*}{$\mathbf{p}$} \\
\hline & $\mathbf{n}$ & $\%$ & $\mathbf{n}$ & $\%$ & \\
\hline Hematoma & 2 & 9,5 & 0 & 0,0 & \\
\hline Epiteliólise & 1 & 4,7 & 0 & 0,0 & \\
\hline Cicatriz hipertrófica & 2 & 9,5 & 1 & 5,0 & \\
\hline Estenose umbilical & 2 & 4,7 & 1 & 5,0 & \\
\hline Total & 10 & 47,6 & 2 & 10,0 & $<0,010^{*}$ \\
\hline
\end{tabular}

* Diferença estatisticamente significante. Teste Exato de Fisher.

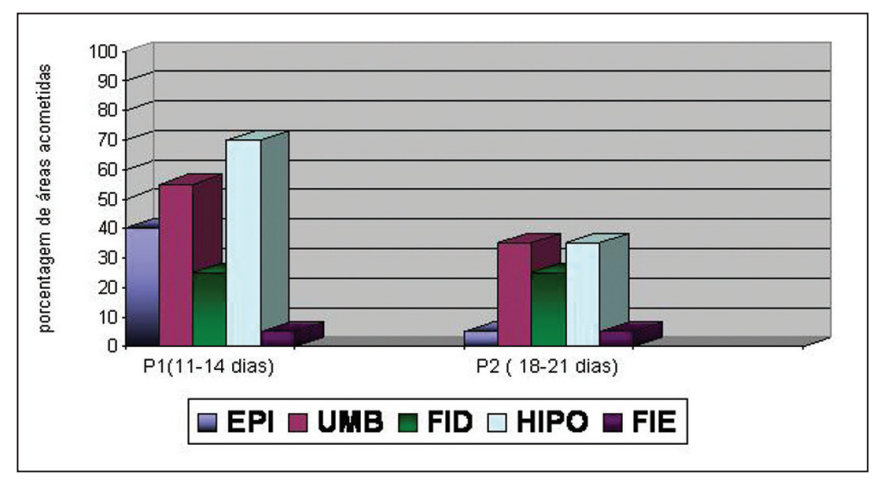

Figura 6 - Áreas acometidas por coleções fluidas nos pacientes do Grupo A (abdominoplastia) nos periodos de 11-14 e 18-21 dias. EPI (epigástrio), UMB (umbilical), FID (fossa ilíaca direita), HIPO (hipogástrio) e FIE (fossa ilíaca esquerda).

dos casos e, ultrassonograficamente, em $38,1 \%$ dos casos. O teste de McNemar demonstrou que os diagnósticos positivos para seroma no grupo A foram significativamente maior com a utilização do ultrassom do que com a análise clínica $(\mathrm{p} \leq$ 0,024). No grupo B (lipoabdominoplastia), no período de 11-14 dias (P1), o diagnóstico clínico de seroma foi realizado em $5 \%$ dos casos e o ultrassonográfico em $10 \%$, não havendo diferença significante entre os dois métodos diagnósticos nesse grupo (Tabela 5).

Comparando-se a presença de coleções fluidas nas cinco diferentes regiões da parede abdominal [epigástrio (EPI), umbilical (UMB), hipogástrio (HIPO), fossa ilíaca direita (FID) e fossa ilíaca esquerda (FIE)], observou-se no grupo A, período de 11-14 dias que as regiões FIE e FID apresentaram presença de coleções fluidas significantemente maiores do que as regiões UMB e HIPO, no entanto não houve diferença significante para o período de 18-21 dias (Figura 6). Mesmo não abrangendo o nível de significância, os resultados sugerem incidência maior de coleções na região FID em

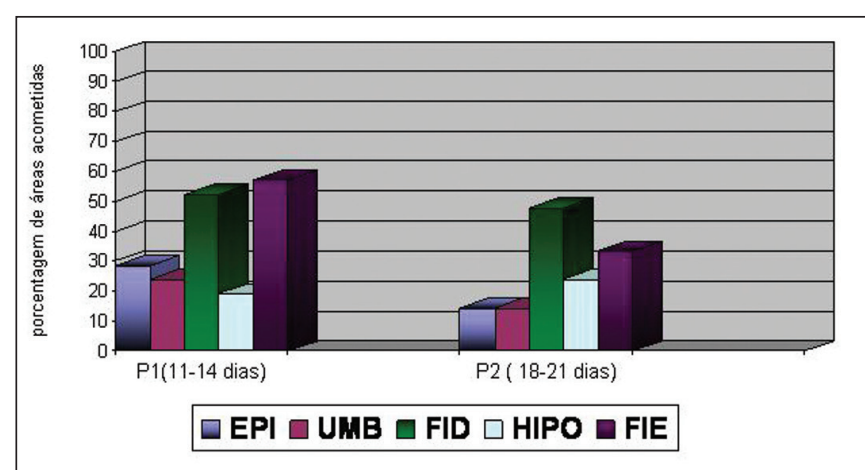

Figura 7-Áreas acometidas por coleções fluidas nos pacientes do Grupo B (lipoabdominoplastia) nos periodos de 11-14 e 18-21 dias. EPI (epigástrio), UMB (umbilical), FID (fossa ilíaca direita), HIPO (hipogástrio) e FIE (fossa ilíaca esquerda). FIE (fossa ilíaca esquerda).

relação às regiões EPI e UMB.

No grupo $\mathrm{B}$, houve diferença significante com maior acúmulo de coleções fluidas na região HIPO em relação às regiões EPI, FID e FIE no período de 11-14 dias e nas regiões UMB e HIPO em relação às regiões EPI e FIE no período de 18-21 dias (Figura 7).

Analisando-se as complicações pós-operatórias, o teste exato de Fisher mostrou que, a porcentagem no grupo A $(47,6 \%)$ foi significativamente maior do que no grupo B (10\%) (Tabela 6).

As Figuras 8 e 9 exemplificam os resultados cirúrgicos obtidos nos pacientes dos grupos A e B, evoluindo sem complicações.

\section{DISCUSSÃO}

O seroma é uma coleção fluida que apresenta características de exsudato com predomínio de neutrófilos e altas 

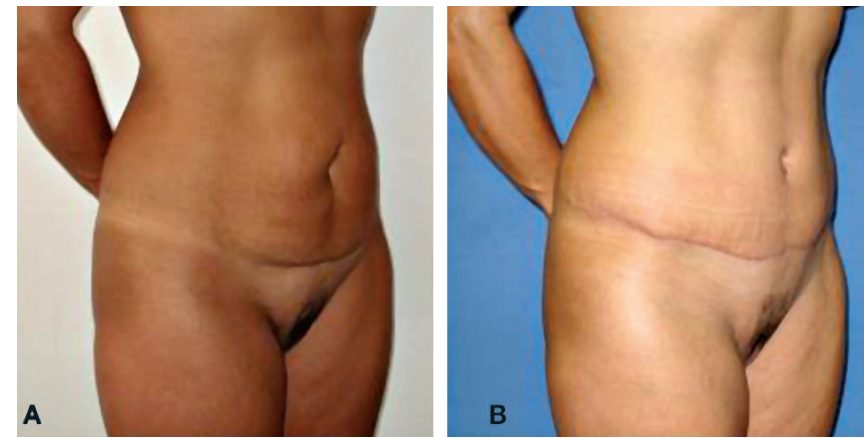

Figura 8-Exemplo de aspecto pré e pós-operatório de abdominoplastia convencional (visão oblíqua direita). A) Préoperatório. Notam-se flacidez cutânea supra e infraumbilical com moderada lipodistrofia abdominal. B) Pós-operatório de 6 meses.

taxas de proteínas ${ }^{20}$. Os mecanismos postulados para sua formação são: 1. Secção de numerosos canais linfáticos, 2. espaço morto decorrente do extenso descolamento do retalho dermogorduroso, 3 . forças de cisalhamento entre o retalho e a aponeurose; 4. liberação de mediadores inflamatórios ${ }^{21,22}$. O acúmulo de seroma sob o retalho abdominal pode levar a aumento da pressão nessa região, causando deiscências, necroses, drenagens espontâneas pela ferida operatória e infecção $0^{23,24}$. A necessidade de múltiplas aspirações causa repetidas visitas ao consultório, aumento dos custos pósoperatórios e, principalmente, desconforto ao paciente ${ }^{25}$. Seromas crônicos não tratados podem levar à formação de uma cápsula fibrosa ao seu redor, denominada pseudobursa. Essa cápsula pode sofrer um processo de contração, evoluindo em alguns casos com deformidade da parede abdominal ${ }^{26}$, sendo indicado tratamento cirúrgico ${ }^{23,24}$.

Os principais fatores predisponentes à formação do seroma são a obesidade ou grandes perdas ponderais (resultam em um sistema linfático hipertrófico), a extensão da área de descolamento do retalho dermogorduroso (devido ao aumento do espaço morto), cicatriz supraumbilical prévia (por funcionar como uma barreira à drenagem linfática) e a associação de lipoaspiração ${ }^{27}$. Kim \& Stevenson ${ }^{28}$ analisaram a formação de seroma em pacientes submetidos à abdominoplastia e observaram que pacientes com sobrepeso ou obesidade apresentaram incidência maior de seroma (38\%) em relação aos pacientes com peso normal (19\%). No presente estudo, a amostra dos dois grupos foi constituída de pacientes com índice de massa corpórea (IMC) inferior a $30 \mathrm{~kg} / \mathrm{m}^{2}$, e não houve diferença significante em relação ao IMC nos grupos.

$\mathrm{O}$ advento da lipoaspiração permitiu melhor tratamento do contorno corporal, principalmente quando associada à abdominoplastia. Matarasso ${ }^{27} \mathrm{e}$ Teimourian ${ }^{29}$ postularam que a incidência de coleções fluidas é maior quando a lipoaspiração é associada à abdominoplastia quando comparada à abdominoplastia como procedimento isolado. Alguns autores
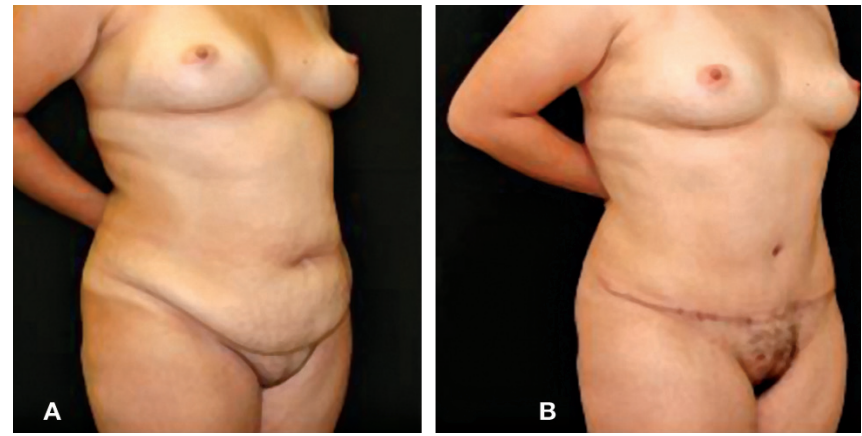

Figura 9 - Exemplo de aspecto pré e pós-operatório de lipoabdominoplastia (visão oblíqua direita). A) Pré-operatório. Notam-se flacidez cutânea supra e infraumbilical com acentuada lipodistrofia abdominal. B) Pós-operatório de 6 meses.

afirmam que a incidência de seroma é de $100 \%$ quando lipoaspiração e abdominoplastia são associadas ${ }^{30}$. Por outro lado, Dillerud ${ }^{12}$, em estudo retrospectivo envolvendo 487 abdominoplastias associadas à lipoaspiração, verificou que apenas $1 \%$ desenvolveu seroma. Khan ${ }^{15}$ não encontrou diferença significante na incidência desta complicação quando comparou pacientes submetidos à abdominoplastia e abdominoplastia associada à lipoaspiração.

Araco et al. ${ }^{31}$ estudaram retrospectivamente 494 pacientes submetidos a abdominoplastia associada à lipoaspiração e verificaram que $4,7 \%$ apresentaram seroma. Os autores demonstraram que o volume lipoaspirado não influenciou na formação de seroma e que o peso do retalho dermogorduroso removido pode ser um importante fator predisponente para sua formação.

Ausência de descolamentos ${ }^{32,33}$ ou descolamento reduzidos $^{14,22,34}$, uso de malhas compressivas no pós-operatório e a utilização dos pontos de adesão ${ }^{35,36}$ são alternativas eficazes na prevenção da formação de seroma nas abdominoplastias.

A análise da literatura demonstra que estudos retrospectivos apresentam incidência menor na formação de seroma em relação aos estudos prospectivos. Isto ocorre provavelmente pelo fato do seroma ser uma complicação menor, nem sempre descrita em alguns prontuários ${ }^{12,15,16,37}$. Na maioria dos estudos, a avaliação da presença do seroma em abdominoplastia é realizada clinicamente, portanto seromas não palpáveis de pequeno volume não são diagnosticados. Entretanto, seromas crônicos de pequeno volume também podem encapsular, formando pseudobursa ${ }^{38}$. Mohammad et al. ${ }^{16}$ demonstraram, por meio de análise com ultrassom, a presença de coleções líquidas após abdominoplastias em $51,8 \%$ dos casos, sendo que em $35,7 \%$ essas coleções eram de pequeno volume e não haviam sido diagnosticadas clinicamente. No grupo submetido à abdominoplastia, houve diferença significante entre o diagnóstico clínico $(23,8 \%)$ e o ultrassonográfico $(38,1 \%)$, o mesmo não ocorrendo no grupo submetido à lipoabdominoplastia. Foi realizada 
análise comparativa entre os dois métodos diagnósticos nos dois grupos apenas no período de 11-14 dias, uma vez que os achados ultrassonográficos desse período poderiam influenciar no diagnóstico clínico no segundo período (18-21 dias). Verificou-se que houve detecção maior dessa complicação utilizando-se ultrassom no grupo abdominoplastia. Isso ocorre pois o exame clínico da parede abdominal pode falhar na detecção de coleções líquidas devido ao edema local e desconforto do paciente durante o exame físico ${ }^{16,17}$.

Como a formação do seroma é um fenômeno que ocorre entre a $2^{\mathrm{a}}$ e a $3^{\mathrm{a}}$ semana de pós-operatório, no presente estudo, realizou-se o exame ultrassonográfico em dois períodos distintos (11-14 e 18-21 dias) com a finalidade de se abranger o período de maior formação dessa coleção ${ }^{3,5,23}$.

A ultrassonografia tem sido o método de escolha para o diagnóstico de seroma pós-abdominoplastias. As características de cada coleção líquida formada podem ser interpretadas e quantificadas pelo radiologista. Seromas são usualmente coleções livres de eco, enquanto hematomas aparecem de maneira mais complexa, com septações ou camadas ${ }^{16}$. A deteç̧ão dessas coleções permite a realização de intervenções precoces, quando necessário.

No presente estudo, observou-se que no grupo lipoabdominoplastia os drenos foram retirados 1,43 dias mais cedo em relação ao grupo abdominoplastia; sendo um aspecto importante, pois a precocidade na retirada dos drenos diminui o desconforto do paciente e a morbidade no pós-operatório relacionado ao seu uso ${ }^{12}$. Borile et al..$^{39}$ e Nahas et al. ${ }^{5}$, em estudos prospectivos, afirmam que o uso de dreno não diminuiu a incidência de seroma, pois como se trata de um fenômeno que ocorre entre a $2^{\mathrm{a}}$ e a $3^{\mathrm{a}}$ semana de pós-operatório, sua incidência não está relacionada ao uso de dreno.

A presença de coleções fluidas não é uma complicação, mas uma evolução habitual após a abdominoplastia. Pequenas coleções fluidas são reabsorvidas sem repercussões no resultado da cirurgia. Embora um limite de coleções que não requerem aspiração não seja bem determinado, o volume de $20 \mathrm{ml}$ foi considerado nesse estudo como o limite máximo a ser tratado conservadoramente (sem aspiração). Embora nem sempre sejam clinicamente detectáveis, coleções maiores que $20 \mathrm{ml}$ podem induzir a formação de cápsula (pseudobursa), com consequente deformidade da parede abdominal. No grupo A, apenas $5(23,8 \%)$ pacientes apresentaram coleções contínuas com volume maior que $20 \mathrm{ml}$ e foram aspirados.

A lipoabdominoplastia diminui a formação de seroma pelos seguintes fatores: 1. Menor descolamento da região supraumbilical, com um espaço morto menor e preservação dos vasos perfurantes dos músculos retos do abdome. 2. Preservação da fáscia de Scarpa e da gordura profunda da região infraumbilical, com manutenção de maior número de vasos linfáticos. Felmerer et al. ${ }^{40}$, em estudo anatômico da região infraumbilical, observaram uma rica rede de vasos linfáticos adjacentes à derme (sistema linfático superficial) e na gordura profunda à fáscia de Scarpa (sistema linfático profundo), fatores que podem ser responsáveis pela redução do seroma. Existem poucos estudos disponíveis na literatura sobre incidência de seroma após lipoabdominoplastia. Heller et al. ${ }^{41} \mathrm{e}$ Saldanha et al. ${ }^{42}$ verificaram clinicamente nas lipoabdominoplastias realizadas incidência de seroma em 3,3\% e $0,4 \%$ dos casos, respectivamente. No presente estudo, verificou-se por análise ultrassonográfica uma incidência de $10 \%$ no P1 (11-14 dias) e 0\% no P2 (18-21 dias) no grupo lipoabdominoplastia.

\section{CONCLUSÃO}

As pacientes submetidas à lipoabdominoplastia apresentaram menor incidência de seroma quando comparadas às submetidas à abdominoplastia convencional nos dois períodos estudados.

\section{REFERÊNCIAS}

1. Sociedade Brasileira de Cirurgia Plástica. Pesquisa Data Folha;2009.

2. van Uchelen JH, Werker PM, Kon M. Complications of abdominoplasty in 86 patients. Plast Reconstr Surg. 2001;107(7):1869-73.

3. Baroudi R, Ferreira CA. Seroma: how to avoid it and how to treat it. Aesthet Surg J. 1998;18(6):439-41.

4. Chaouat M, Levan P, Lalanne B, Buisson T, Nicolau P, Mimoun M. Abdominal dermolipectomies: early postoperative complications and long-term unfavorable results. Plast Reconstr Surg. 2000;106(7):1614-8.

5. Nahas FX, Ferreira LM, Ghelfond C. Does quilting suture prevent seroma in abdominoplasty? Plast Reconstr Surg. 2007;199(3):1060-4.

6. Grazer FM, Goldwyn RM. Abdominoplasty assessed by survey with emphasis on complications. Plast Reconstr Surg. 1977;59(4): 513-7.

7. Baxter RA. Controlled results with abdominoplasty. Aesthetic Plast Surg. 2001;25(5):357-64.

8. Illouz YG. Une nouvelle technique pour les lipodystrophies localis. Rev Rev Chir Esth Franc. 1980;6(9):9-14.

9. Avelar JM. Fat suction versus abdominoplasty. Aesthetic Plast Surg. 1985;9(4):265-75.

10. Wilkinson TS, Swartz BE. Individual modifications in body contour surgery: the "limited" abdominoplasty. Plast Reconstr Surg. 1986;77(5):779-84

11. Hakme F. Technical details in the lipoaspiration associated to abdominoplasty. Rev Bras Cir. 1985;75(5):331-7.

12. Dillerud E. Abdominoplasty combined with suction lipoplasty: a study of complications, revisions, and risk factors in 487 cases. Ann Plast Surg. 1990;25(5):333-8.

13. Saldanha OR, Pinto EB, Matos WN Jr, Lucon RL, Magalhães F, Bello EM. Lipoabdominoplasty without undermining. Aesthet Surg J. 2001;21(6):518-26.

14. Saldanha OR, De Souza Pinto EB, Mattos WN Jr, Pazetti CE, Lopes Bello EM, Rojas Y, et al. Lipoabdominoplasty with selective and safe undermining. Aesthetic Plast Surg. 2003;27(4):322-7.

15. Khan UD. Risk of seroma with simultaneous liposuction and abdominoplasty and the role of progressive tension suture. Aesthetic Plast Surg. 2008;32(1):93-9.

16. Mohammad JA, Warnke PH, Stavraky W. Ultrasound in the diagnosis and management of fluid collection complications following abdominoplasty. Ann Plast Surg. 1998;41(5):498-502.

17. Stocchero IN. Ultrasound and seromas. Plast Reconstr Surg. 
1993;91(1):198.

18. Nahas FX. A pragmatic way do treat abdominal deformities based on skin and subcutaneous excess. Aesthetic Plast Surg. 2001;25(5):365-71.

19. Nahas FX. An aesthetic classification of the abdomen based on the myoaponeurotic layer. Plast Reconstr Surg. 2001;108(6):1787-95.

20. Andrades P, Prado A. Composition of postabdominoplasty seroma. Aesthetic Plast Surg. 2007;31(5):514-8.

21. Kulber DA, Bacilious N, Peters ED, Gayle LB, Hoffman L. The use of fibrin sealant in the prevention of seromas. Plast Reconstr Surg. 1997;99(3):842-9.

22. Hafezi F, Nouhi A. Safe abdominoplasty with extensive liposuctioning. Ann Plast Surg. 2006;57(2):149-53.

23. Keramidas EG, Rodopoulou S, Khan U. Pseudo-cyst formation after abdominoplasty combined with liposuction: A case report and review of the literature. Eur J Plast Surg. 2006;28:400-2.

24. Ersek RA, Schade K. Subcutaneous pseudobursa secondary to suction and surgery. Plast Reconstr Surg. 1989;85(3):442-5.

25. Roje Z, Roje Z, Karanovic N, Utrobicic I. Abdominoplasty complications: a comprehensive approach for the treatment of chronic seroma with pseudobursa. Aesthetic Plast Surg. 2006;30(5):611-5.

26. Zecha PJ, Missotten FE. Pseudocyst formation after abdominoplasty: extravasations of Morel-Lavalleé. Br J Plast Surg. 1999;52(6):500-2.

27. Matarasso A. Liposuction as an adjunct to a full abdominoplasty. Plast Reconstr Surg. 1995;95(5):829-36.

28. Kim J, Stevenson TR. Abdominoplasty, liposuction of the flanks, and obesity: analyzing risk factors for seroma formation. Plast Reconstr Surg. 2006;117(3):773-9.

29. Teimourian B. Management of seroma in abdominoplasty. Aesthet Surg J. 2005;25(5):510-1.

30. Armand L, Hunstad J, Saltz R, Toledo L. Panel discussion: issues in abdominoplasty. Aesthetic Surg J. 2000;20:403-9.
31. Araco A, Gravante G, Araco F, Sorge R, Cervelli V. Postoperative seromas after abdominoplasty: a retrospective analysis of 494 patients and possible risk factors. Plast Reconstr Surg. 2009;123(4):158e-9e.

32. Illouz YG. Anew safe and aesthetic approach to suction abdominoplasty. Aesthetic Plast Surg. 1992;16(3):237-45.

33. Avelar JM. Abdominoplasty: a new technique without undermining and fat layer removal. Arq Catarinense Méd. 2000;29:147-9.

34. Graf R, de Araujo LR, Rippel R, Neto LG, Pace DT, Cruz GA. Lipoabdominoplasty: liposuction with reduced undermining and traditional abdominal skin flap resection. Aesthetic Plast Surg. 2006;30(1):1-8.

35. Pollock H, Pollock T. Progressive tension sutures: a technique to reduce local complications in abdominoplasty. Plast Reconstr Surg. 2000;105(7):2583-6.

36. Mladick RA. Progressive tension suture to reduce complications in abdominoplasty. Plast Reconstr Surg. 2001;107(2):619.

37. Pitanguy I, Salgado F, Murakami R, Radwanski HN, Mauad Junior R. Abdominoplasty: classification and surgical techniques. Rev Bras Cir. 1995;85(1):23-44.

38. Zimman OA, Butto CD, Ahualli PE. Frequency of seroma in abdominal lipectomies. Plast Reconstr Surg. 2001;108(5):1449-51.

39. Borile G, Pavelecini M, Dreher R, Chem E, Chem RC. The use of suction drains in abdominal dermolipectomy: a randomized clinical trial. Plast Reconstr Surg. 2008;121(4):228e-9e.

40. Felmerer G, Muehlberger T, Berens von Rautenfeld D, Vogt PM. The lymphatic system of the deep inferior epigastric artery perforator flap: an anatomical study. Br J Plast Surg. 2002;55(4):335-9.

41. Heller JB, Teng E, Knoll BI, Persing J. Outcome analysis of combined lipoabdominoplasty versus conventional abdominoplasty. Plast Reconstr Surg. 2008;121(5):1821-9.

42. Saldanha OR, Federico R, Daher PF, Malheiros AA, Carneiro PR, Azevedo SF, et al. Lipoabdominoplasty. Plast Reconstr Surg. 2009;124(3):934-42.

Correspondência para:

Marcello Di Martino

Rua Alexandre Dumas, 1300 /134 - Chácara

Sto. Antonio - São Paulo, SP, Brasil - CEP 04717-003

E-mail: marcelloplast@hotmail.com 